\title{
Impacto de la pandemia por COVID-19 en la epidemiología, manejo y supervivencia de los pacientes con fractura de cadera.
}

\author{
DOI: http//dx.doi.ORG/10.37315/SOTOCAV20212865650 \\ CLIMENT-PERIS VJ, PAULOS DOS SANTOS F, PÉREZ GINER R, OJEDA PEÑA M. \\ SERVICIO DE CIRUGÍA ORTOPÉDICA Y TRAUMATOLOGÍA DEL HOSPITAL “VERGE DELS LLIRIS” D’ALCOI.
}

\begin{abstract}
Resumen
La pandemia por COVID-19 obliga a reorganizar los recursos sanitarios hacia la atención de los pacientes con síndrome respiratorio grave. Mientras la incidencia de patología traumática se redujo no fue así con las fracturas de cadera, pudiendo verse comprometido su manejo. El objetivo de este estudio es evaluar las consecuencias de la pandemia sobre la epidemiología, manejo y resultados de la fractura de cadera. Material y métodos: Estudio observacional de cohortes retrospectivo en el que se incluyeron pacientes con fractura de cadera atendidos entre marzo de 2019 y febrero de 2021 , estableciéndose dos grupos para su comparación, antes y después del inicio de la pandemia. Se registraron las variables demográficas, comorbilidades, antecedentes de fractura por fragilidad y fractura de cadera, tipo de fractura y tipo de tratamiento realizado, demora quirúrgica, duración de la estancia hospitalaria y resultado de PRC para SARS-CoV2 al ingreso. Se analizó la tasa de mortalidad intrahospitalaria y la supervivencia al mes y a los 6 meses. Resultados: Se incluyeron 251 pacientes, 137 (54,58 \%) grupo PRE-COVID y 114 (45,42\%) grupo POST-C. No hubo diferencias en las variables demográficas, comorbilidades, tipo de fractura ni tratamiento realizado. La incidencia absoluta se redujo un 16,8 $\%$ y la incidencia relativa se redujo en un 17,6\%, siendo esta diferencia significativa durante el periodo de confinamiento y en los meses de enero y febrero, periodo de incidencia máxima de COVID-19. La incidencia de PCR + al ingreso fue del $1,75 \%$. No se encontraron diferencias significativas en la demora quirúrgica, estancia y mortalidad hospitalaria, supervivencia al mes ni a los 6 meses. Conclusión: Con la pandemia por COVID-19 se han observado reducciones puntuales de la incidencia de fracturas de cadera, sin cambios en su epidemiología, indicadores de manejo hospitalario ni en la supervivencia de los pacientes al mes y a los 6 meses.
\end{abstract}

\section{Summary}

The COVID-19 pandemic has forced a reorganization of health resources towards the care of patients with severe respiratory syndrome. While the incidence of traumatic pathology was reduced, this was not the case with hip fractures, and their management could be compromised. The objective of this study is to evaluate the consequences of the pandemic on the epidemiology, management and outcomes of hip fracture. Material and methods: A retrospective observational cohort study was done including patients with hip fracture treated between March 2019 and February 2021 , establishing two groups for comparison, before and after the onset of the pandemic. Demographic variables, comorbidities, history of fragility fracture and hip fracture, type of fracture and type of treatment performed, surgical delay, length of hospital stay, and PCR result for SARS-CoV2 upon admission were recorded. In-hospital mortality rate and survival at 1 and 6 months were analyzed. Results: 251 patients were included, 137 (54.58\%) PRE-COVID group and 114 (45.42\%) POST-C group. There were no differences in demographic variables, comorbidities, type of fracture, or treatment performed. The absolute incidence was reduced by $16.8 \%$ and the relative incidence was reduced by $17.6 \%$, this difference was significant during lockdown period and in the months of January and February, the period of maximum incidence of COVID-19. The incidence of PCR + on admission was $1.75 \%$. No significant differences were found in surgical delay, hospital stay and mortality, survival at one month or at 6 months. Conclusion: With the COVID-19 pandemic, specific reductions in the incidence of hip fractures have been observed, without changes in its epidemiology, indicators of hospital management and in patient survival at one and 6 months.

Palabras clave: hip fracture, covid-19, pandemic. SARS-COV2, epidemiology.

\section{Correspondencia:}

Vicente J Climent Peris

E Mail: vicentcp@gmail.com 


\section{INTRODUCCIÓN}

La irrupción de la infección por el virus SARS-CoV-2 origina una situación de emergencia sanitaria sin precedentes, siendo declarada la situación de pandemia por la OMS el 11 de marzo de $2020^{1}$. En España se declaró el estado de alarma el 14 de marzo de 2020, iniciando un periodo de confinamiento domiciliario estricto que se mantuvo hasta el 11 de mayo cuando se inició un periodo de desescalada progresiva. Las consecuencias para el sistema sanitario fueron inmediatas, con el colapso de todos los niveles asistenciales y la necesidad de dirigir los recursos para hacer frente a la altísima demanda asistencial derivada del síndrome respiratorio agudo originado por el coronavirus. Esta situación llevó a la cancelación de los procedimientos de cirugía ortopédica programada y por otra parte a la reducción de la incidencia de patología traumática, sin embargo parece que la incidencia de fractura de cadera no se afectó de la misma manera, manteniéndose constante ${ }^{2}$. Diversos estudios han puesto de manifiesto el incremento de mortalidad en los pacientes con fractura de cadera e infección por COVID-19 $9^{3-6}$. La evolución de la pandemia sigue un patrón en oleadas de incrementos de contagios, con periodos de saturación del sistema sanitario lo que origina un detrimento en los recursos dedicados a otras patologías como la fractura de cadera, pudiendo afectar al manejo hospitalario o al seguimiento posterior de estos pacientes.

El objetivo de este estudio fue analizar los cambios que la pandemia por COVID-19 pudiera originar en la epidemiología y en diversos aspectos relacionados con el manejo de los pacientes con fractura de cadera en el departamento de salud de Alcoi: incidencia, tipo de fractura, demora quirúrgica, duración de la hospitalización y la incidencia de la infección por SARS-COV2, y por otra parte estudiar el impacto de la pandemia sobre la supervivencia de estos pacientes.

\section{MATERIAL Y MÉTODO}

Se realizó un estudio observacional de cohortes retrospectivo en el que se incluyeron pacientes con diagnóstico de fractura de cadera atendidos en el servicio de urgencias de nuestro hospital entre el marzo de 2019 y febrero de 2021. Se utilizaron los criterios de inclusión del Registro Nacional de Fracturas de Cadera $^{7}$ : fractura por fragilidad (caída desde la propia altura), edad igual o mayor a 75 años y entender y firmar el documento de consentimiento informado para participar en el estudio (el paciente o familiar cercano). Siguiendo el mismo criterio se excluyeron las fracturas producidas por mecanismos de alta energía y las fracturas patológicas de origen neoplásico. EI periodo de seguimiento post-fractura se estableció en 6 meses.

Se establecieron dos grupos de pacientes para su comparación: aquellos que ingresaron antes del 1 de marzo de 2020 que formaron el grupo pre-COVID (PRE-C) y aquellos que ingresaron a partir de dicha fecha, que formaron el grupo post-COVID (POST-C).
En todos los casos se registraron las variables demográficas (edad, sexo), comorbilidades (índice de Charlson, ASA), antecedentes de fractura por fragilidad y fractura de cadera, tipo de fractura y tipo de tratamiento realizado, demora quirúrgica y duración de la estancia hospitalaria. Se identificaron aquellos casos con PRC positiva para SARS-CoV2 en el momento del ingreso. Se analizó la tasa de mortalidad intrahospitalaria y se realizó un análisis de supervivencia post-operatoria al mes y a los 6 meses. También se calcularon las curvas de supervivencia de Kaplan-Meyer para cada grupo

Se utilizó el test de Kolmogorov-Smirnof para determinar la normalidad de la distribución las variables cuantitativas. Los valores obtenidos para las variables cualitativas se expresaron como valores absolutos y porcentajes; las variables cuantitativas como media y desviación estándar o mediana y rango intercuartil según el tipo de distribución.

Para la comparación entre los grupos se utilizó la prueba Chi cuadrado de Pearson para las variables categóricas y la prueba $t$ de Student o $U$ de Mann-Whitney para las variables cuantitativas según su distribución. Las curvas de Kaplan-Meyer se compararon mediante la prueba de Mantel-Cox. Se consideró el p-valor <0,05 como estadísticamente significativo.

El análisis se llevó a cabo con el software IBM SPPS Stadistics v25.

\section{RESULTADOS}

Se incluyeron en el estudio un total de 251 pacientes de los cuales 137 (54,58 \%) formaron el grupo PRE-C y 114 $(45,42 \%)$ el grupo POST-C. El seguimiento fue de 6 meses para todos los pacientes. Las características demográficas y comorbilidades de los grupos se recogen en la tabla I. No se encontraron diferencias estadísticamente significativas en cuanto a dichas variables entre los dos grupos.

\begin{tabular}{|c|c|c|c|}
\hline & PRË-C & POST-C & $p$-valor \\
\hline$N^{\circ}$ de casos (n) & 137 & 114 & \\
\hline Edad & $85,48(5,51)$ & $86,13(4,67)$ & $0,32^{*}$ \\
\hline Sexo (\%) & & & $0,82^{\star \star}$ \\
\hline Varón & $39(28,5 \%)$ & $31(27,2 \%)$ & \\
\hline Mujer & $98(71,5 \%)$ & $83(72,8 \%)$ & \\
\hline Indice Charlson & $6(2)$ & $6(2)$ & $0,38^{\star * \pi \star}$ \\
\hline ASA & & & $0,56^{\approx \star}$ \\
\hline I & $0(0 \%)$ & $1(0,9 \%)$ & \\
\hline II & $50(36,5 \%)$ & $42(36,8 \%)$ & \\
\hline III & $86(62,8 \%)$ & $71(63,3 \%)$ & \\
\hline IV & $1(0,7 \%)$ & $0(0 \%)$ & \\
\hline Fractura Previa & $26(19 \%)$ & $25(21,9 \%)$ & $0,56^{\star \star}$ \\
\hline Fr Cadera Previa & $14(10,2 \%)$ & $11(9,6 \%)$ & $0,54^{* \star}$ \\
\hline \multicolumn{4}{|l|}{ " $t$-Student } \\
\hline \multicolumn{4}{|l|}{ ** Chi-cuadrado } \\
\hline$* * *$ U Man-Withney & & & \\
\hline
\end{tabular}

Tabla I: Variables demográficas y comorbilidades

La incidencia absoluta de fractura de cadera se redujo un $16,8 \%$ en el periodo COVID; la incidencia relativa pasó de $100,78 \times 10^{5}$ habitantes a $83,08 \times 10^{5}$ habitantes, lo que significó una reducción del $17,7 \%$. Analizando la 
distribución mensual de los casos, la incidencia media mensual fue de 11,42 (DE 5,63) en el grupo PRE-C y 8,75 (DE 4,2) en el grupo POST-C, sin diferencia estadística $(p=0,35)$; se observó una reducción significativa de la incidencia en los meses de abril y mayo, coincidiendo con la instauración del confinamiento domiciliario, así como,en los meses de enero y febrero, los de máxima incidencia de ingresos hospitalarios por COVID-19 en nuestro departamento de salud $(p=0,029)$.

Analizando el tipo de fractura no se encontraron diferencias significativas entre los grupos ( $p=0,5)$. El 97,81\% y $100 \%$ de los pacientes de los grupos PRE-C y POST-C fueron tratados quirúrgicamente; no se encontraron diferencias significativas en cuanto al tipo de procedimiento realizado $(p=0,28)$. El tipo de fractura de los pacientes de cada grupo y el tipo de tratamiento realizado se recogen en la tabla II.

\begin{tabular}{c|ccc}
\multicolumn{1}{c}{ PRE-C } & POST- C & p-valor \\
\hline Tipo Fractura & $87(63,5 \%)$ & $77(67,5 \%)$ & $0,503^{*}$ \\
Extracapsular & $50(36,5 \%)$ & $37(32,5 \%)$ & \\
Intracapsular & & & \\
Tipo Tratamiento & & $279^{*}$ \\
Clavo endomedular & $85(62 \%)$ & $77(67,5 \%)$ & \\
DHS & $1(0,7 \%)$ & $0(0 \%)$ & \\
T Canulados & $2(1,5 \%)$ & $1(0,9 \%)$ & \\
Prótesis Parcial & $43(31,4 \%)$ & $36(31,6 \%)$ & \\
Prótesis Total & $3(2,2 \%)$ & $0(0 \%)$ & \\
TTO no Quirúrgico & $3(2,2 \%)$ & $0(0 \%)$ & \\
& & & \\
* Chi-cuadrado & & &
\end{tabular}

Tabla II. Tipo de fractura y tratamiento realizado.

La demora quirúrgica media fue de 3,66 (DE 2,56) y 3,38 (DE 1,68) días y la estancia hospitalaria de 7,72 (DE 4,12) y 6,82 (DE 2,71) para los grupos PRE-C y POST-C respectivamente. Se apreció mayor número de valores atípicos y extremos en la duración de la hospitalización en el grupo PRE-C (Fig. 1) y una reducción de la estancia media en 1,1 días en el grupo POST-C, aunque las diferencias observadas no resultaron ser estadísticamente significativas para la demora quirúrgica ni la estancia hospitalaria $(p>0,05)$.

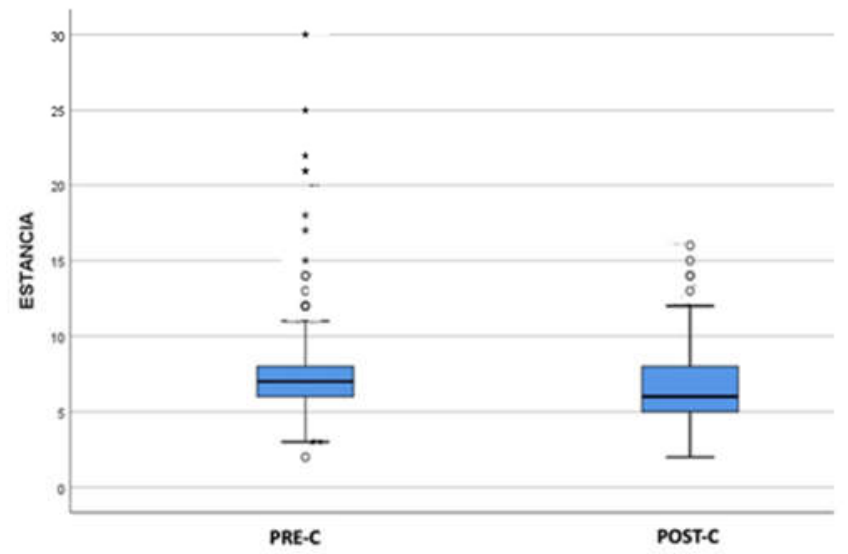

Figura 1. Duración de la estancia hospitalaria. La mediana es 7 y 6 para los grupos PRE-C y POST-C respectivamente (rango intercuartil 3), en el primero de ellos se aprecia mayor número de valores atípicos y extremos.
La mortalidad durante el ingreso hospitalario fue de 5 (3,6 $\%)$ y $6(5,2 \%)$ pacientes respectivamente en los grupos PRE-C y POST-C. Se observó un incremento del 1,6\% en el segundo grupo, pero esta diferencia no resultó ser significativa $(p=0,53)$.

La supervivencia fue del $87,6 \%$ y $87,7 \%$ al mes de la fractura en los grupos PRE-C y POST-C respectivamente $(p=0,97)$, siendo $77,4 \%$ y $76,3 \%$ a los 6 meses $(p=0,84)$. Las curvas de supervivencia de Kaplan-Meyer se muestran en la figura 2, no existiendo diferencias significativas entre ambas $(p=0,87)$.

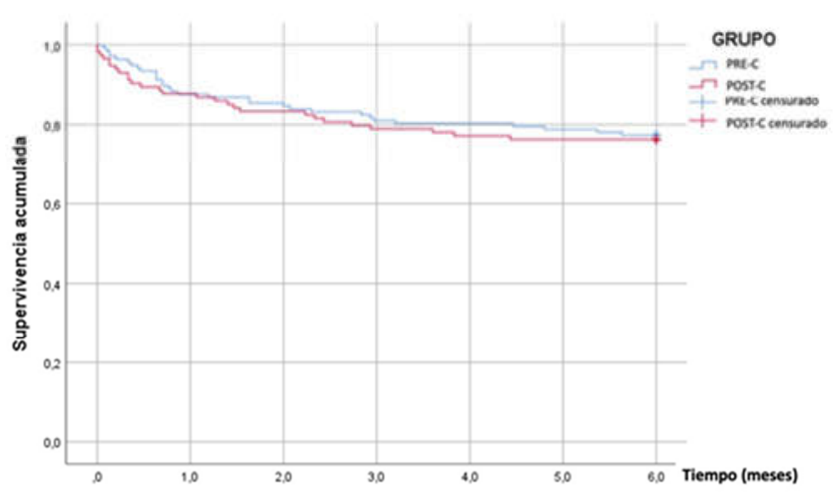

Figura 2. Curvas de Kaplan-Meier para cada grupo; Mantel Cox $p=0,87$

Dos pacientes del grupo POST-C (1,75\%) presentaron PCR + para COVID-19 en el momento del ingreso; ambos pacientes estaban vivos a los 6 de seguimiento.

\section{DISCUSIÓN}

Salvo reducciones puntuales de la tasa de incidencia, el primer año de la pandemia por COVID-19 ha tenido escaso impacto sobre la epidemiología, manejo y resultados del proceso de fractura de cadera en nuestro departamento. La incidencia de fractura de cadera se redujo en un $57 \%$ en el departamento de salud de Alcoi durante el periodo de confinamiento domiciliario, al inicio de la pandemia. Se han publicado tasas de reducción variables en nuestro país que van desde una reducción del $30 \%^{8}$ a no observar cambios en la misma ${ }^{2,9}$. En países de nuestro entorno se ha comunicado una tendencia similar, con reducciones entre el 11 y el $13 \%$ en Francia $^{10}$, $28 \%$ en Portugal ${ }^{11}$ o del $20 \%$ en Irlanda ${ }^{12}$. Las características demográficas de los pacientes no han cambiado con la pandemia, encontrándonos que alrededor del $70 \%$ son mujeres, con edad media entorno a los 85 años; tampoco se ha modificado el grado de comorbilidad de los pacientes con una mediana de 6 para el índice de Charlson y ASA III o peor en más del $60 \%$ de los casos. Estos datos difieren de los publicados por LeBrun et al. ${ }^{13}$ que observaron un incremento de los índices comorbilidad de los pacientes atendidos al inicio de la pandemia respecto al mismo periodo de 2019 o los 
obtenidos en una de las series más largas en Reino Unido con reducción significativa de pacientes ASA III e incremento de ASA $\mathrm{IV}^{14}$, sin diferencias en las variables demográficas en ninguno de estos estudios. La modificación de las pautas de convivencia originadas por la pandemia, que se inició con el confinamiento domiciliario y siguió con la reducción de los contactos sociales, no ha modificado la incidencia de los distintos patrones de fractura y tampoco las indicaciones quirúrgicas en estos pacientes: alrededor del $60 \%$ fueron fracturas extracapsulares que fueron tratadas mediante osteosíntesis endomedular.

Es importante analizar cuál ha sido la repercusión de la saturación de los hospitales y la movilización de los recursos hacia el tratamiento de los pacientes con patología respiratoria grave sobre la atención a los pacientes con fractura de cadera. La demora media para llevar a cabo la intervención quirúrgica no cambió en nuestro hospital en relación con la pandemia, situándose alrededor de 72 horas en ambos grupos. Esta demora se debió fundamentalmente a factores como la toma de antiagregantes y anticoagulantes o la falta de disponibilidad de quirófanos durante los fines de semana. Estas circunstancias no se modificaron en el grupo POST-C por lo que no parece que los cambios organizativos a nivel hospitalario influyeran en la demora hasta la intervención. La estancia hospitalaria media se redujo en 1,1 días, con reducción de valores atípicos y extremos en el grupo POST-C. Esto nos indica que se optimizó el manejo de los pacientes para conseguir el alta precoz de los mismos. Tampoco se demostró una repercusión significativa sobre la mortalidad durante el ingreso, que se incrementó un 1,76\%. Varios estudios comparativos analizados muestran una reducción significativa de la demora hasta la intervención y de la duración de la estancia hospitalaria durante la pandemia respecto al grupo control ${ }^{12,14-16}$.

El análisis de los cambios en la supervivencia de los pacientes después de sufrir una fractura de cadera es otro aspecto que refleja el impacto de la infección por COVID-19 concurrente con la fractura por una parte y el impacto del colapso de los diversos niveles asistenciales en el seguimiento post-operatorio de estos pacientes.

La incidencia de PCR COVID-19 positiva en nuestro grupo POST-C fue de 2 pacientes $(1,75 \%)$ lo que no permite analizar su impacto en la supervivencia. Grassi et al ${ }^{17}$ observaron en Italia, durante los dos primeros meses de la pandemia, una incidencia de la infección en los pacientes con fractura de cadera del $14 \%$, incrementándose cuatro veces la mortalidad, que alcanzó el $35 \%$ a los 45 días. En la serie publicada por Egol et al $^{18}$ la mortalidad fue del $53 \%$ al mes en los pacientes COVID + (5,6 \% en COVID -). En un metanálisis que incluyó 560 casos de fractura de cadera COVID + la mortalidad alcanzó el 36,61 \% frente al 8,6 \% en los 306 casos COVID - incluidos ${ }^{19}$. Resultados similares obtuvieron Fessler et $\mathrm{al}^{20}$ y Alcock $^{3}$ en sendos metanálisis.

La mortalidad al mes de nuestros pacientes no se modificó con la pandemia, siendo del $12 \%$, ascendiendo a los 6 meses al 23 y $24 \%$ para los grupos PRE-C y POST-C respectivamente. Un estudio realizado en el Reino Unido confirmó un incremento significativo de la mortalidad a los 30 días $(16,3 \%)$ respecto al observado antes de la pandemia $(4,2-9,8 \%)$, pero su incidencia de pacientes COVID + fue del $28 \%{ }^{21}$. En el estudio ya comentado de Egol et al. la mortalidad al mes se incrementó durante la pandemia del 2,7 a $12,3 \%{ }^{18}$. Dallari et $\mathrm{al}^{22}$ en un estudio multicéntrico en el que se incluyeron 1390 pacientes con fractura de cadera atendidos en 14 hospitales de Italia durante la primera ola de la pandemia encontraron una mortalidad al mes del $23 \%$ en COVID + y del $5 \%$ en COVID -. Los resultados del metanálisis de Alcock et $\mathrm{al}^{3}$ muestran que no hay diferencia en la mortalidad precoz en pacientes COVID - durante la pandemia si se comparan con el mismo periodo anterior a la pandemia (riesgo relativo $1.0995 \%$ IC $0.69-1.73)$.

Parece demostrado que la infección concomitante por COVID-19 constituye un importante factor de riesgo de mortalidad durante el primer mes tras la fractura de cadera, no observando cambios en los pacientes no contagiados que sufrieron dicha fractura durante la pandemia.

Observamos en nuestra serie una menor supervivencia al mes que en los estudios analizados; esto podría deberse a que se incluyeron pacientes de edad igual o superior a 75 años, a diferencia de otros estudios que incluían a pacientes de 65 años o más. La tasa de mortalidad al mes recogida en el registro nacional de fracturas de cadera de España, que sigue el mismo criterio de edad, fue del 8,3\% en 2019 y del $10,1 \%$ en $2020^{23,24}$, cifras que, aunque siendo inferiores, se aproximan más a los de nuestra serie. Este dato negativo en cuanto a supervivencia en el grupo PRE-C que se mantiene sin empeorar de forma significativa en el grupo POST-C nos indicaría que en nuestro departamento la evolución post-operatoria de los pacientes con fractura de cadera depende poco de los recursos sanitarios disponibles en ese momento en los distintos niveles asistenciales (atención especializada, atención domiciliaria, rehabilitación, atención primaria) y que existen posibilidades de mejora en la atención a estos pacientes.

El presente estudio tiene varias limitaciones: se trata de un estudio retrospectivo, no se ha valorado es estado funcional y de dependencia de los pacientes antes y después de la fractura, que podría verse influenciado también por el confinamiento y las restricciones de movilidad impuestas durante la pandemia. El número limitado de pacientes no nos permite estudiar los efectos de la infección por COVID19 y no se ha analizado la incidencia de complicaciones post-operatorias que en el contexto de la pandemia podría demorarse su diagnóstico y tratamiento y contribuir al mal pronóstico del paciente.

\section{Como conclusiones podemos decir que:}

La pandemia por COVID-19 ha originado una reducción puntual de la incidencia de fracturas de cadera que coincide con los momentos de mayor presión asistencial a nivel hospitalario por pacientes con sintomatología respiratoria grave. 
No se han producido otros cambios en cuanto a la epidemiología de esta fractura, no observando cambios en la edad, distribución por sexos ni tipo de fractura.

No se han observado cambios en los parámetros relativos a la atención hospitalaria como son la demora, quirúrgica, estancia o mortalidad intrahospitalaria.
- $\quad$ No se han detectado cambios en la supervivencia de los pacientes al mes ni a los 6 meses.

- $\quad$ Estos resultados ponen de manifiesto la necesidad de incrementar los recursos que, en condiciones epidemiológicas normales, se dedican a la atención de los pacientes con fractura de cadera.

\section{Bibliografía}

1. World Health Organization. Mar 11. 2020. Coronavirus disease 2019 (COVID-19) situation report-51.On line. Disponible en: https://www.who.int/docs/default-source/coronaviruse/situation-reports/20200311-sitrep-51-covid-19.pdf?sfvrsn=1ba62e57_10.

2. Nuñez JH, Sallent A, Lakhani K, Guerra-Farfan E, Vidal N, Ekhtiari S, et al. Impact of the COVID-19 Pandemic on an Emergency Traumatology Service: Experience at a Tertiary Trauma Centre in Spain. Injury 2020; 51:1414-8.

3. Alcock H, Moppett EA, Moppett IK. Early mortality outcomes of patients with fragility hip fracture and concurrent SARS-CoV-2 infection : a systematic review and meta-analysis. Bone Jt open 2021; 2(5):314-22.

4. Fell A, Malik-Tabassum K, Rickman S, Arealis G. Thirty-day mortality and reliability of Nottingham Hip Fracture Score in patients with COVID19 infection. J Orthop 2021; 26:111-4.

5. Dupley L, Oputa TJ, Bourne JT, Lum J, Hodhody G, Rogers S, et al. 30-day mortality for fractured neck of femur patients with concurrent COVID-19 infection. Eur J Orthop Surg Traumatol 2021; 31(2).

6. Oputa TJ, Dupley L, Bourne JT. One Hundred Twenty-Day Mortality Rates for Hip Fracture Patients with COVID-19 Infection. Clin Orthop Surg 2021; 13(2):135-43.

7. Sáez-López P, González-Montalvo JI, Ojeda-Thies C, Mora-Fernández J, Muñoz-Pascual A, Cancio JM, et al. Spanish National Hip Fracture Registry (SNHFR): a description of its objectives, methodology and implementation. Rev Esp Geriatr Gerontol 2018; 53(4):188-95.

8. González-Martín D, Álvarez-De la Cruz J, Martín-Vélez P, Boluda-Mengod J, Pais-Brito JL, Herrera-Pérez M. Quantitative and qualitative analysis of the influence of confinement by COVID-19 in fracture patients entered in a traumatology service at a third level hospital. Rev Esp Cir Ortop Traumatol 2021; 65(5):374-81.

9. Miranda I, Sangüesa-Nebot MJ, González A, Doménech J. Impact of strict population confinement on fracture incidence during the COVID-19 pandemic. Experience from a public Health Care Department in Spain. J Orthop Sci 2021; Available from: /pmc/articles/PMC8081314/

10. Paccou J, Lenne X, Ficheur G, Theis D, Cortet B, Bruandet A. Analysis of Hip Fractures in France during the First COVID-19 Lockdown in Spring 2020. JAMA Netw Open 2021; 4(11):1-11.

11. Mazeda C, Santos PB, Vilas-Boas P, Antão J, Barcelos A. What happened to hip fragility fractures during COVID-19 pandemic? Acta Reumatol Port 2021; 46(3):252-6.

12. Crozier-Shaw G, Hughes AJ, Conlon B, Sheehan E, Merghani K. Hip fracture care during Covid-19: a regional trauma centre's experience. Ir J Med Sci 2021; 190(4): 1275-80.

13. LeBrun DG, Konnaris MA, Ghahramani GC, Premkumar A, Defrancesco CJ, Gruskay JA, et al. Hip fracture outcomes during the COVID-19 pandemic: Early results from New York. J Orthop Trauma 2020; 3 4(8): 403-10.

14. Wignall A, Giannoudis V, De C, Jimenez A, Sturdee S, Nisar S, et al. The impact of COVID-19 on the management and outcomes of patients with proximal femoral fractures: a multi-centre study of 580 patients. J Orthop Surg Res 2021; 16(1).

15. Nazemi AK, Al-Humadi SM, Tantone R, Hays TR, Bowen SN, Komatsu DE, et al. Hip Fractures Before and During the COVID19 Pandemic: Comparative Demographics and Outcomes. Geriatr Orthop Surg Rehabil 2021;12: 21514593211003077

16. Orfanos G, Al Kaisi K, Jaiswal A, Lim J, Youssef B. The effect of COVID-19 pandemic on the care of fragility hip fracture patients in the United Kingdom. A case control study in a major trauma centre. Surgeon 2021; 19(6): e440-5.

17. Grassi A, Andriolo L, Golinelli D, Tedesco D, Rosa S, Gramegna P, et al. Higher 90-Day Mortality after Surgery for Hip Fractures in Patients with COVID-19: A Case-Control Study from a Single Center in Italy. Int J Environ Res Public Health 2021;18(10).

18. Egol KA, Konda SR, Bird ML, Dedhia N, Landes EK, Ranson RA, et al. Increased mortality and major complications in hip fracture care during the COVID-19 pandemic: A New York city perspective. J Orthop Trauma. 2020; 34(8): 395-402.

19. Kumar Jain V, Lal H, Kumar Patralekh M, Vaishya R. Fracture management during COVID-19 pandemic: A systematic review. J Clin Orthop Trauma 2020;11(Suppl 4):S431-41. 
20. Fessler J, Jacobsen T, Lauritzen JB, Jørgensen HL. Mortality among hip fracture patients infected with COVID-19 perioperatively. Eur J Trauma Emerg Surg 2021; 47(3): 659-64.

21. Thakrar A, Chui K, Kapoor A, Hambidge J. Thirty-Day Mortality Rate of Patients with Hip Fractures during the COVID-19 Pandemic: A Single Centre Prospective Study in the United Kingdom. J Orthop Trauma 2020; 34(9): E325-9.

22. Dallari D, Zagra L, Cimatti P, Guindani N, D'Apolito R, Bove F, et al. Early mortality in hip fracture patients admitted during first wave of the COVID-19 pandemic in Northern Italy: a multicentre study. J Orthop Traumatol 2021; 22(1).

23. López SP, Thies OC, Campelo G, Sierra PT. Registro Nacional de Fracturas de Cadera. Informe Anual 2019. (On line). Disponible en: http://rnfc.es/wp-content/uploads/2021/03/Informe-Anual-RNFC-2019_digital-1.pdf

24. Informe anual RNFC 2020. (On line). Disponible en: http://rnfc.es/wpcontent/uploads/2021/05/Informe-2020-por-hospitales.pdf 